\title{
Experimental Study of Wake / Flap Interaction Noise and the Reduction of Flap Side Edge Noise
}

\author{
Florence V. Hutcheson* \\ NASA Langley Research Center, Hampton, VA \\ Daniel J. Stead ${ }^{* *}$ \\ Northrop Grumman, Hampton, VA \\ Gerald E. Plassman^ \\ National Institute of Aerospace, Hampton, VA
}

The effects of the interaction of a wake with a half-span flap on radiated noise are examined. The incident wake is generated by bars of various widths and lengths or by a simplified landing gear model. Single microphone and phased array measurements are used to isolate the effects of the wake interaction on the noise radiating from the flap side edge and flap cove regions. The effects on noise of the wake generator's geometry and relative placement with respect to the flap are assessed. Placement of the wake generators upstream of the flap side edge is shown to lead to the reduction of flap side edge noise by introducing a velocity deficit and likely altering the instabilities in the flap side edge vortex system. Significant reduction in flap side edge noise is achieved with a bar positioned directly upstream of the flap side edge. The noise reduction benefit is seen to improve with increased bar width, length and proximity to the flap edge. Positioning of the landing gear model upstream of the flap side edge also leads to decreased flap side edge noise. In addition, flap cove noise levels are significantly lower than when the landing gear is positioned upstream of the flap mid-span. The impact of the local flow velocity on the noise radiating directly from the landing gear is discussed. The effects of the landing gear side-braces on flap side edge, flap cove and landing gear noise are shown.

\section{Introduction}

During airport approach and landing, the aircraft landing gear system and deployed flaps are major sources of airframe noise. Significant efforts have been dedicated towards developing quieter landing gear and flap side edge designs, but the noise resulting from the landing gear wake interaction with the flaps also needs to be addressed. A limited number of experimental studies ${ }^{1,2}$ have been performed to date to examine the noise resulting from such interaction. The most detailed acoustic study that has been reported was performed by Oerlemans and Pott-Pollenske ${ }^{1}$. In their experiment, the authors examined the noise resulting from the interaction of a wake with a full span flap. The wake was produced by a simplified landing gear model with and without the presence of a landing gear cavity. The landing gear, rather than the cavity, was found to be the dominant contributor to the interaction noise, which was shown to radiate from the leading edge of the flap and scale with the $6^{\text {th }}$ power of velocity. Increasing the distance between the landing gear and the flap and the use of a porous flap leading edge were also found to lead to a reduction of the interaction noise.

In the present study, the effects of the interaction of a wake with a half-span flap on the radiated noise were examined. Particular attention was given to the noise resulting from the wake interaction with the flap side edge. The incident wake was generated either by simple blunt bodies (bars) or by a simplified landing gear model. The test objectives were as follows: (1) determine the interaction noise sensitivity to the wake generator placement and geometry; (2) assess the potential of wake generators for the reduction of flap side edge noise; (3) identify where the landing gear should be placed to minimize the noise.

\footnotetext{
* Senior Research Engineer, Aeroacoustics Branch, Senior Member AIAA

** Senior Engineer

^Associate Principal Engineer, Senior Member AIAA
} 


\section{Test Models and Experimental Set-up}

This experiment was conducted at NASA Langley Research Center in the Quiet Flow Facility (QFF). The QFF is an anechoic facility equipped with a 2 by 3 foot rectangular nozzle. Side plates attached to the 2 -foot sides of the nozzle are used to support models above the nozzle, while the 3-foot sides of the test section remain open. The test model used in the present experiment is a 16-inch chord, 3-foot span NACA $63_{2}$-215 main element airfoil with a halfspan Fowler flap of 4.8-inch chord. The main airfoil was positioned in the test section at an angle of attack of $16^{\circ}$ such that the local aerodynamics properly represented high-lift device conditions on the flap and in the main element aft region. ${ }^{3}$ Streamlined U-brackets were used to position the flap. Strips of serrated tape ( 0.004 " thick) were placed along the span of the main airfoil to trip the boundary layer and induce its transition to a turbulent state. The strips were positioned along the airfoil leading edge (LE), along the model pressure and suction sides at $9 \%$ chord, and along the model pressure side at 27\% chord. The test model is shown installed in the QFF test section in Figure 1.

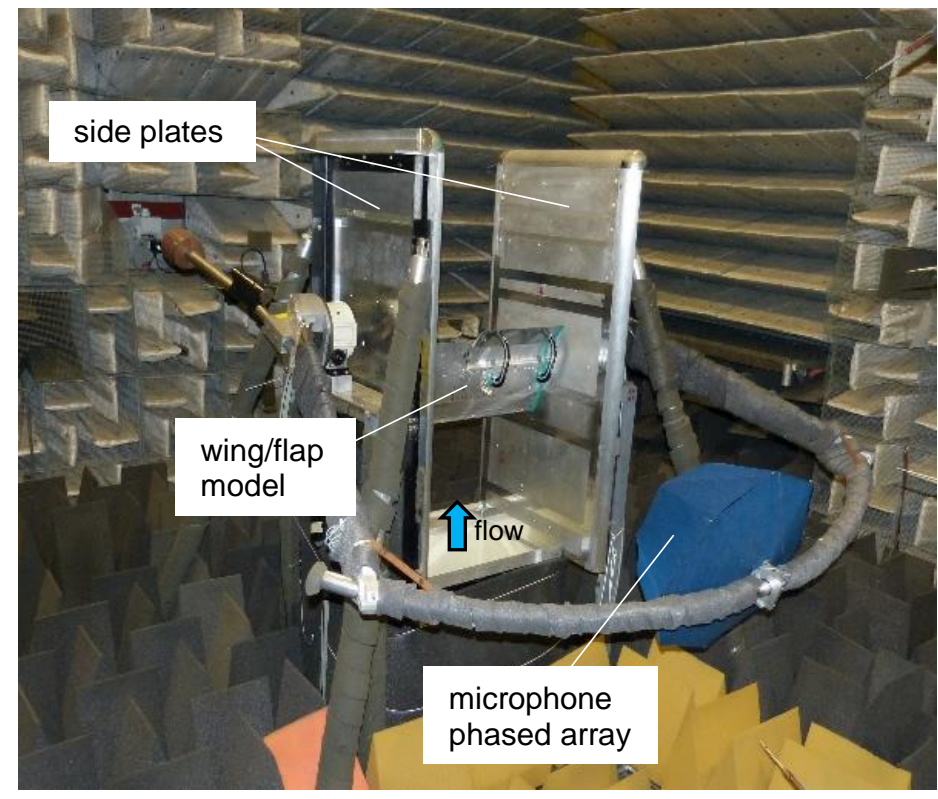

Figure 1. Airfoil/flap model installed in the test section of the Quiet Flow Facility.

In the first part of this study, the interaction noise from the wake produced by simple bluff body geometries was examined. The bluff bodies consisted of bars of various widths and lengths. Referring to Figure 2(a), the bars' widths and lengths were, respectively, as follows: bar\#1: 1" x 6"; bar\#2: 0.7" x 6"; bar\#3: 1" x 2"; bar\#4: 0.5" x 6"; bar\#5: 0.7 " $x$ 8.5". Bar\#2 is shown mounted to the main airfoil, upstream of the flap side edge in Figure 2(b).

(a)

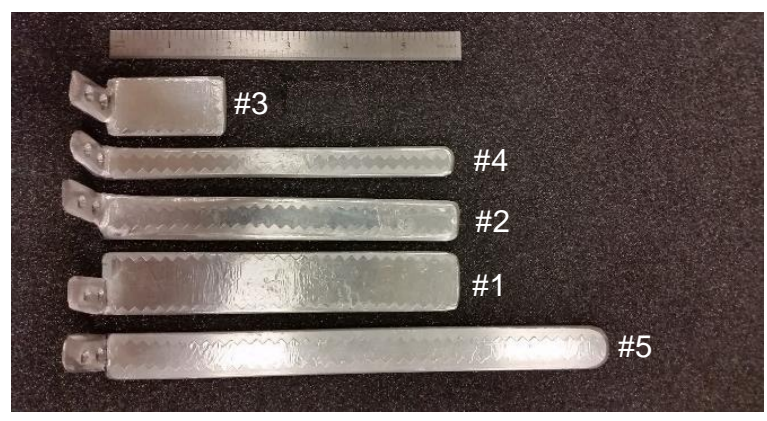

(b)

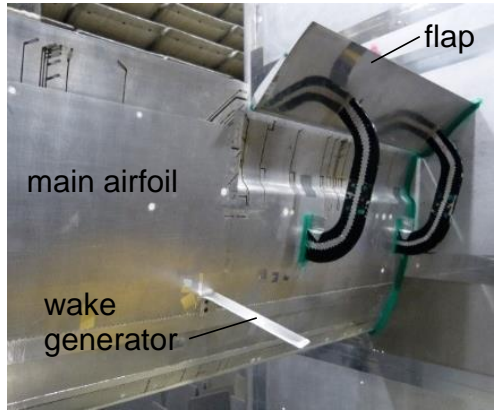

Figure 2. (a) Wake generators (bars); (b) Main airfoil with half-span flap installed in test section with bar\#2 mounted upstream of flap side edge. 
In the second part of the study, the interaction noise from the wake produced by a simplified landing gear model was examined. The landing gear model (shown in Figure 3(a)) was composed of a main strut, a door, two side-braces, and a truck with six wheels. These components were removable so as to allow for an evaluation of their respective effects on the wake/flap interaction noise. Because it was determined that the wake from the wheels and truck portion of the landing gear would not impinge on the airfoil/flap model, and hence not directly contribute to the interaction noise, the gear model was solely tested with the truck and wheels removed (as shown in Figure 3(b)). This determination was performed through a simple visualization of the flow streamlines by placing a thin string into the flow at different locations around the installed model. Although the absence of the truck and wheels assembly might result in slightly modified velocities around neighboring gear components, that effect was deemed secondary to the elimination of a significant landing gear noise source. The remaining landing gear components were treated to further minimize direct noise radiation from the landing gear, since landing gear noise was not a focus of this study. Thus, the trailing edges of the landing gear door and side-braces were extended (as seen in Figure 3(b)) with a thin strip of 0.005 -inch thick aluminum tape. This was done to eliminate trailing edge bluntness noise ${ }^{4}$ by creating the effect of sharp trailing edges. The noise radiating from the landing gear main strut was also reduced by distributing strips of plastic tie-wrap (also shown in Figure 3(b)) along the length of the strut to render the vortex shedding less coherent ${ }^{5}$.

(a)

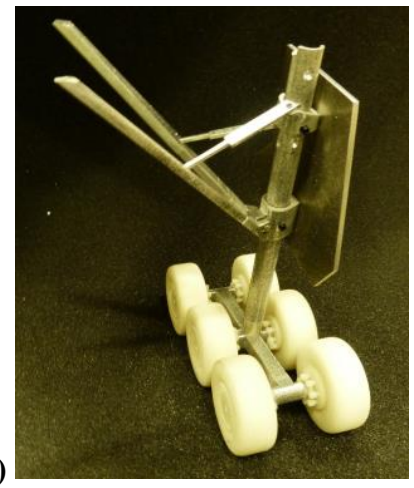

(b)

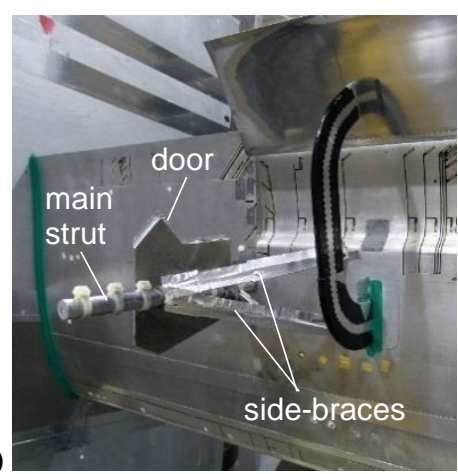

Figure 3. (a) Simplified landing gear model; (b) Landing gear model (truck \& wheels removed) mounted upstream of the flap side edge.

The QFF's Medium Aperture Directional Array (MADA), consisting of 41 B\&K 1/8" microphones projecting from an acoustically treated frame, was used to acquire the acoustic data. The array was positioned on the model pressure side, in the mid-span plane, at $90^{\circ}$ to the main airfoil's chord and 5 feet from the main airfoil trailing edge. A screen of thin fabric was installed over the face of the array to prevent wind gusts from increasing microphone selfnoise. High- and low-pass filters were set, respectively, at $70 \mathrm{~Hz}$ and $50 \mathrm{kHz}$, and 1,000 non-overlapping segments of $2^{13}$ data points were acquired at a sample rate of $142.85 \mathrm{kHz}$.

Although noise measurements were performed for flow speeds ranging from Mach (M) 0.11 to 0.17 and for two flap deployment angles $\left(29^{\circ}\right.$ and $\left.39^{\circ}\right)$, the results presented in this paper focus on the highest Mach number and flap deployment angle tested, as those best represent the wing/flap configurations and flow conditions that would be observed during aircraft landing. The effects of flow Mach number and flap deployment angle are only discussed for a subset of bars and landing gear configurations.

\section{Test Results:}

\subsection{Noise Radiation from the Wing/Flap Model: Baseline Case}

The DAMAS ${ }^{6}$ method was used for the processing of the microphone phased array data to obtain spatial distributions of the noise sources for the wing/flap model in the absence of wake generators (baseline case). The DAMAS noise source distributions obtained at a flow Mach number of 0.17 , for the $39^{\circ}$ flap configuration, are presented in Figure 4. Results are shown for sets of bands that sum four standard one-third octave frequency bands. Spanwise and chordwise coordinates shown are with respect to a 50" high and 50" wide planar grid (scanning grid used for the DAMAS processing) positioned through the main element trailing edge, parallel to the face of the array. This places the grid along the chordline of the main airfoil. The profile of the test model shown is as seen by the center 
of the phased array. The results presented were obtained by first generating DAMAS maps for each narrowband (7 bins combined) frequency. The narrowband results were then summed (on a pressure-squared basis) to obtain onethird octave band maps from which four bands were themselves summed to obtain each data frame shown in Figure 4. The center frequencies of the first and last one-third octave bands included in each sum are indicated. It is seen from Figure 4 that the strongest noise sources are concentrated in the side edge region of the flap. This is clearly seen in the noise maps covering the $3.15 \mathrm{kHz}$ to $40 \mathrm{kHz}$ frequency range. Examination of the DAMAS maps obtained for individual one-third octave bands revealed that below the $2 \mathrm{kHz}$ one-third octave band, the DAMAS processing could only marginally resolve the noise source distributions due to the relatively large beamwidth (hence low resolution) of the MADA in that frequency range.

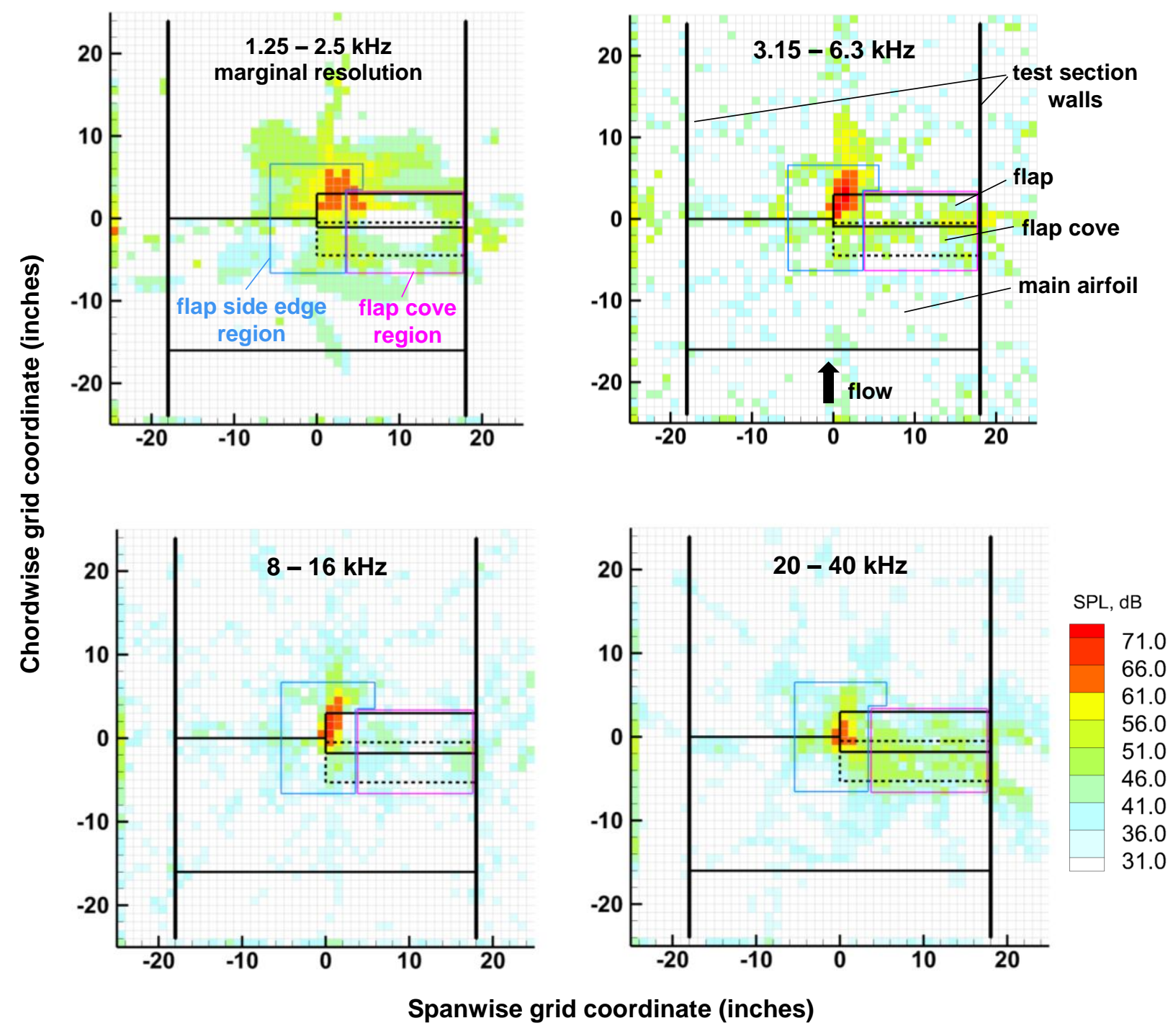

Figure 4. DAMAS noise source distribution maps in summed one-third octave bands for the baseline model configuration (i.e., no wake generators); $39^{\circ}$ flap; $M=0.17$.

The corresponding one-third octave band spectra of the noise radiating from the flap side edge and cove regions of the model are presented in Figure 5. These spectra are obtained from the DAMAS maps generated for each onethird octave band. The spectral level for a given one-third octave frequency band is obtained by summing the mean squared pressures over the region of interest. The flap side edge and cove regions are defined in Figure 4. The flap side edge region encloses the side edge of the flap, as well as the part of the cove that is closest to the side edge, while 
the flap cove region encloses the rest of the flap and cove. It is seen in Figure 5 that the noise radiating from the flap side edge clearly dominates the spectra for all frequencies and that the noise radiating from the flap cove region is most prominent in the low frequency range around $3.15 \mathrm{kHz}$, as well as at high frequencies near $40 \mathrm{kHz}$. The sharp increase in cove noise observed in the high frequency range was shown in Reference 7 to be strongly related to noise that is scattered from the trailing edge of the main airfoil and channeled into the flap cove. Modification of the main airfoil trailing edge (by placing a 0.5 " long serrated tape extension along the portion of the trailing edge spanning the cove region) to reduce the strength and coherence of the vorticity emanating from it was found to decrease this cove noise high frequency peak.

The auto-spectrum from an individual microphone of the MADA as well as the total DAMAS spectrum are also included in Figure 5. The levels of the total DAMAS spectrum are determined by summing the mean squared pressures over the entire region covered by the scanning grid (borders excluded). It is seen that the single microphone and total DAMAS spectra are in good agreement and that they both provide a good representation of the noise radiating from the flap side edge above $2 \mathrm{kHz}$ (where flap side edge noise dominates). Both capture the high frequency spectral peak produced from the flap cove region. Although the DAMAS processing could only marginally resolve the noise source distributions between 1 and $2 \mathrm{kHz}$, the dominant noise sources appeared to remain in the general vicinity of the flap side edge. Thus, the single microphone auto-spectrum may also provide an approximate representation of the flap side edge noise between 1 and $2 \mathrm{kHz}$, as long as flap side edge noise continues to dominate the spectra in that frequency range. Below $1 \mathrm{kHz}$, noise source distributions could not be identified.

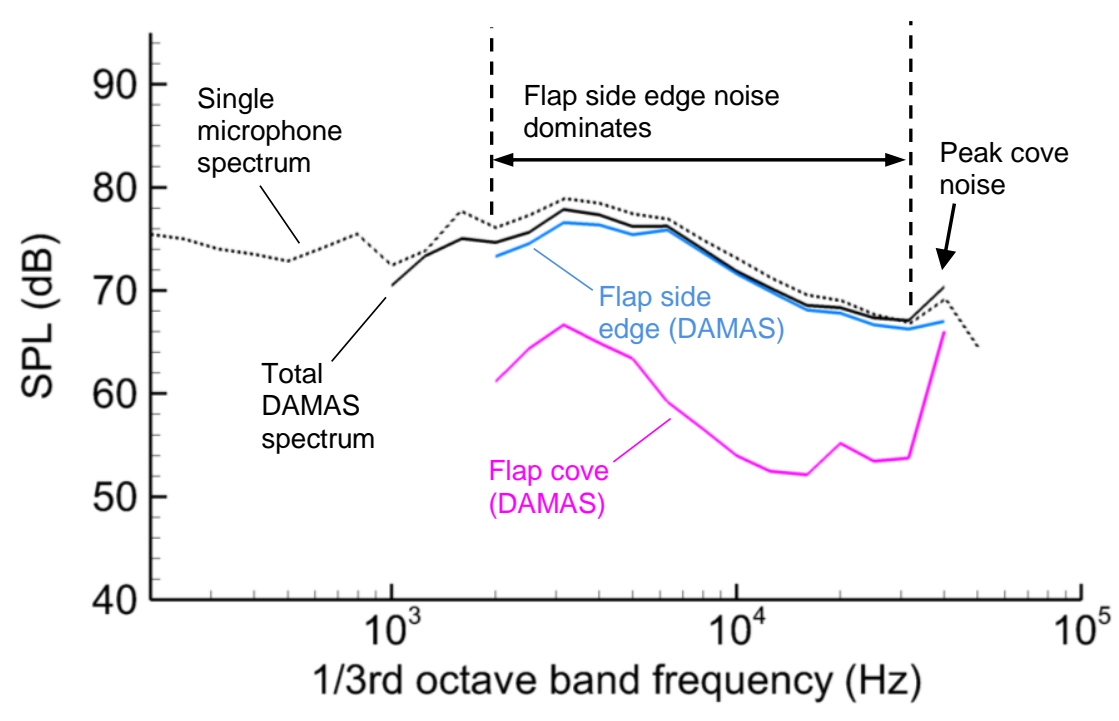

Figure 5. One-third octave band spectra of overall, flap side edge and flap cove noise. Baseline model configuration; $39^{\circ}$ flap; $M=0.17$.

\subsection{Bar Wake / Flap Interaction}

The interaction noise from the wake produced by a simple bluff body, bar\#2, was examined first. The bar was positioned on the main airfoil, upstream of the flap, at different spanwise and streamwise stations. Single microphone noise spectra obtained with bar\#2 installed alternatively upstream of the flap mid-span and upstream of the flap side edge are shown in Figure 6 (along with a sketch depicting the aforementioned bar positions). The noise spectrum obtained in the absence of the bar (baseline case) is also included for reference. Compared to the baseline spectrum, installation of the bar inboard of the flap leads to a small increase in noise across most of the frequency range, while placing the bar upstream of the flap side edge leads to a decrease in noise between $2 \mathrm{kHz}$ and $12.5 \mathrm{kHz}$ (and to an increase in noise below and above that frequency range). Furthermore, changes in the bar's streamwise station (from $67 \%$ to $36 \%$ chord) on the main airfoil leave the noise spectra nearly unchanged when the bar is inboard of the flap; however, the frequency range over which noise reduction is achieved when the bar is positioned upstream of the flap side edge is reduced. The increase in noise observed in the very low frequency range when the bar is moved further upstream of the flap side edge will be discussed later in the paper. 

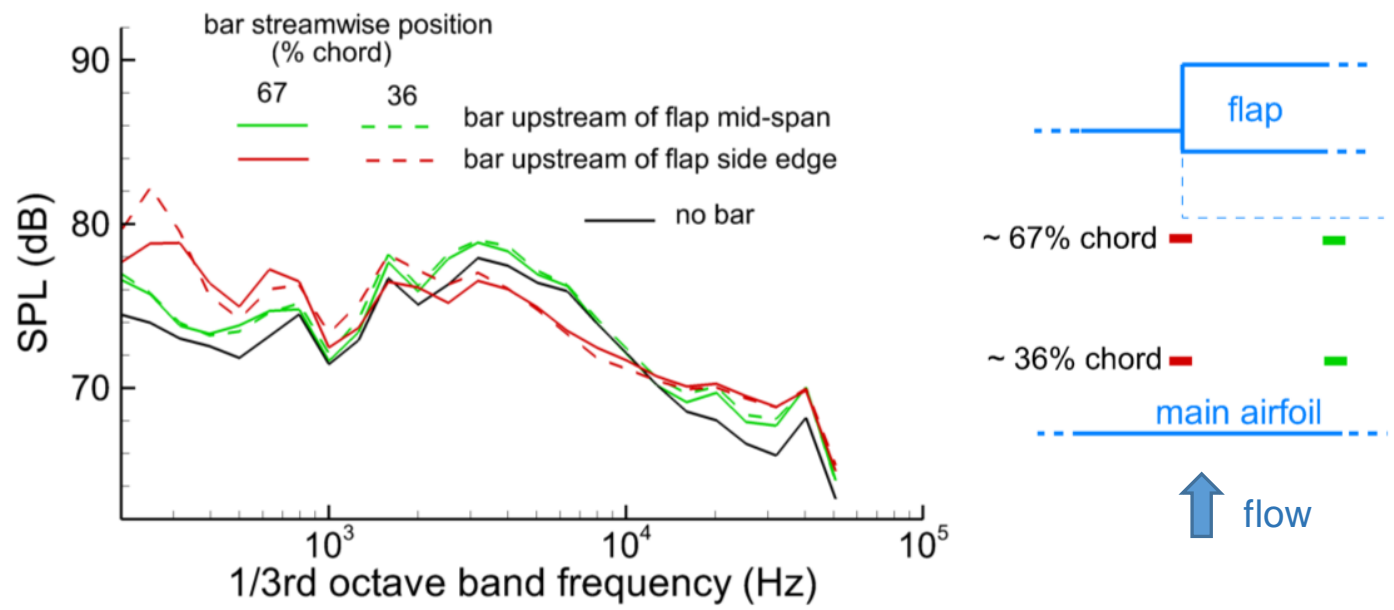

Figure 6. One-third octave band spectra from single microphone measurements. Bar\#2; $39^{\circ}$ flap; $M=0.17$.

The spectra of the noise radiating, respectively, from the flap side edge and flap cove regions (defined in Figure 4) are presented in Figure 7 for the same test cases. These spectra were obtained through DAMAS processing in the manner described in section 3.1. When compared to the baseline noise spectra (obtained from the wing/flap model in the absence of a wake generator), it is seen that positioning the bar inboard of the flap does not affect the noise radiating from the flap side edge region but leads to a 6 to $8 \mathrm{~dB}$ increase in the noise radiating from the flap cove region. Conversely, positioning the bar upstream of the flap side edge has little effect on the noise radiating from the flap cove region; however, it leads to a 2 to $3 \mathrm{~dB}$ increase in the noise radiating from the flap side edge above $10 \mathrm{kHz}$, as well as a 2 to $3 \mathrm{~dB}$ reduction of the peak flap side edge noise level between 2.5 and $10 \mathrm{kHz}$. Positioning the bar inboard of the flap also raises the level of the $40 \mathrm{kHz}$ cove noise peak above flap side edge noise levels. Changing the streamwise position of the bar is shown in Figure 7(b) to have little effect on cove noise levels. This may indicate that when the bar is moved upstream, the broadening of the wake that occurs (which for constant-strength vortices would be expected to lead to increased noise levels) is compensated by a reduction in the intensity of the vortices entering the flap cove region.

(a)

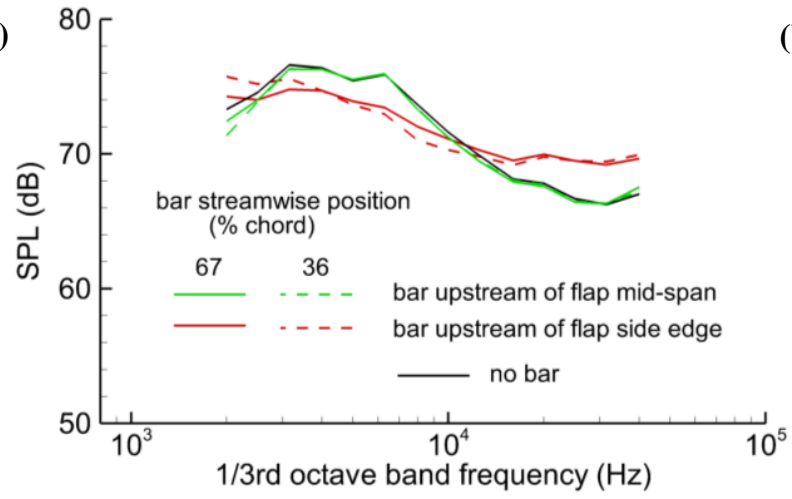

(b)

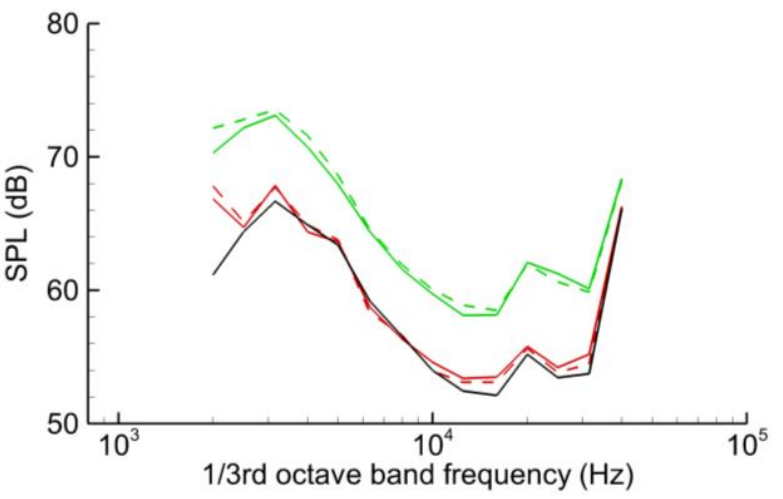

Figure 7. One-third octave band spectra of (a) noise radiating from the flap side edge region and (b) noise radiating from the flap cove region. Bar\#2; $39^{\circ}$ flap; $M=0.17$.

A comparison of the flap side edge noise and cove noise spectra with single microphone spectra (overall noise) is displayed in Figure 8 for the test cases where the bar is positioned upstream of the flap side edge. In these test cases, the noise radiating from the flap side edge region clearly dominates the overall spectra between 2 and $31.5 \mathrm{kHz}$ (as in the baseline case). Therefore, changes in flap side edge noise between 2 and $31.5 \mathrm{kHz}$ are expected to be reflected by 
the same (or very similar) changes in the single microphone spectral levels. This is verified in Figure 8 where the change in flap side edge noise produced by a variation of the bar streamwise station is properly captured by the single microphone measurements. Single microphone spectra are used below to further investigate the effect of wake/flapside-edge interaction on the noise radiating from the flap side edge.

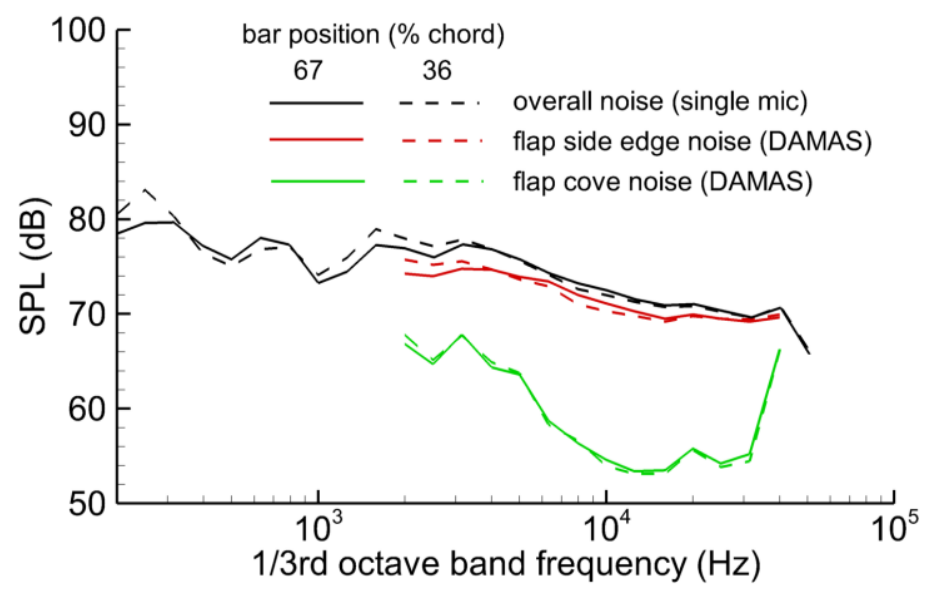

Figure 8. One-third octave band spectra of the overall noise and of the noise radiating from the flap side edge and flap cove regions; Bar\#2 is positioned upstream of the flap side edge at two different streamwise stations; $39^{\circ}$ flap; $M=0.17$.

The effect of the streamwise distance between the bar and the flap side edge on the radiated noise was examined in more detail by positioning bar\#2 directly upstream of the flap side edge (at $92 \%$ chord) and incrementally moving the bar further upstream. The single microphone spectra obtained for each bar streamwise station are displayed in Figure 9. The spectrum obtained for the baseline configuration (no bar) is also shown.

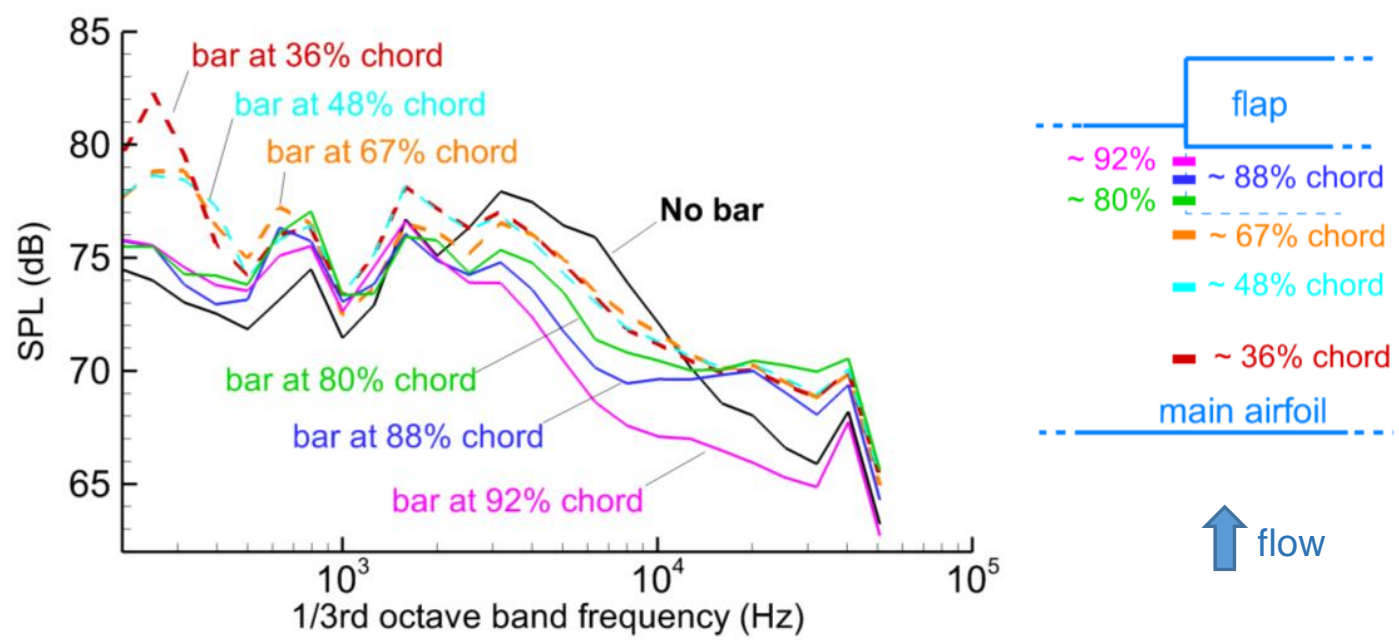

Figure 9. One-third octave band spectra from single microphone measurements. Variation of wake generator streamwise placement with respect to the flap side edge. $B a r \# 2 ; 39^{\circ}$ flap; $M=0.17$.

It is seen from Figure 9 that up to $7 \mathrm{~dB}$ in noise reduction is achieved when the wake generator is placed in the position closest to the flap side edge. With this wake generator placement, noise reduction is achieved over a broad frequency range, namely 2 to $31.5 \mathrm{kHz}$, which (as was shown earlier) is dominated by the noise radiating from the flap side edge. It is also seen that the flap side edge noise reduction benefit decreases as the bar is progressively moved upstream from $92 \%$ chord to $67 \%$ chord. With the bar positioned further upstream, beyond $67 \%$ chord, the noise reduction benefit remains nearly unchanged, and a very low- frequency "hump" develops around $250 \mathrm{~Hz}$. The source of this 
spectral "hump" is unclear. One hypothesis is that it is produced by the generation of quasi-periodicity in the interaction of the flap with the wake and eddies shed. Thus, with the bar positioned at the most upstream position of $36 \%$ chord, the "hump" is replaced by a broad "tone" whose peak frequency scales with changes in velocity (as is shown later in Figure 11). Regarding the reduction in noise that results from the wake/flap-side-edge interaction, recall that flap side edge noise is generated by instabilities in the vortex system that are formed at the flap side edge as well as in the strong shear layer that originates on the pressure side of the flap edge ${ }^{7,8,9}$. The wake produced by the bar introduces a velocity deficit as well as coherent and incoherent flow structures into the flap side edge vortex system forming region, which may be weakening that vortex system and associated instabilities, and hence reducing the radiated noise. These results demonstrate that simple wake generators may be used for the reduction of flap side edge noise.

The effect of the bar geometry on noise reduction is presented next. Bars of different widths and lengths were alternatively positioned upstream of the flap side edge at $92 \%$ chord. The noise spectra obtained for bars of the same length but different widths are shown in Figure 10(a). It is seen that the maximum level of noise reduction achieved increases with the bar width, from $4 \mathrm{~dB}$ with bar\#4, to $7 \mathrm{~dB}$ with bar\#2, and to $8 \mathrm{~dB}$ with bar\#1. Although the noise reduction occurred over a slightly different frequency range with each bar, the peak flap side edge noise level was consistently reduced. The improved noise reduction benefit obtained with the increased bar width continued to be observed as the bar was moved upstream, away from the flap side edge; however, it was also accompanied by a larger increase in low and high frequency noise.
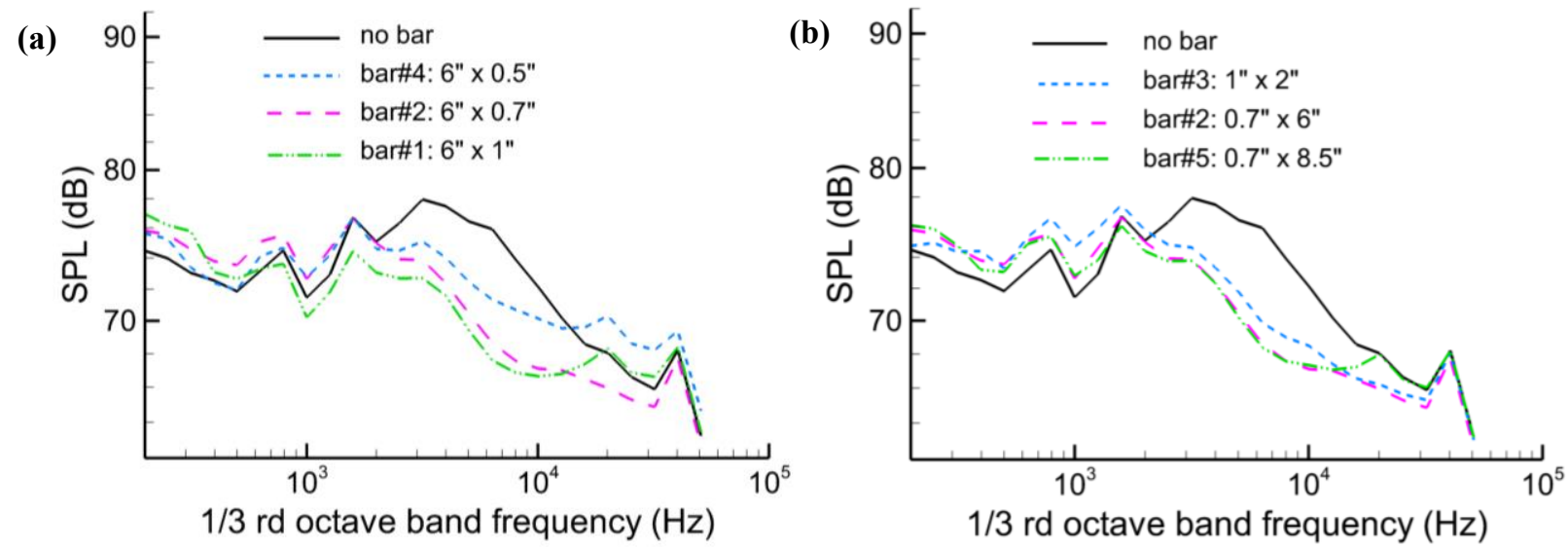

Figure 10. One-third octave band spectra from single microphone measurements; (a) variation of bar width; (b) variation of bar length. Bars are positioned upstream of flap side edge at $92 \%$ chord; $39^{\circ}$ flap; $\mathrm{M}=0.17$.

Regarding the effect of the bar length on the flap side edge noise, it is seen in Figure 10(b) that the noise reduction achieved with the shortest bar, bar\#3, is 1 to $2 \mathrm{~dB}$ less than that achieved with the longer bars. This was also observed when the bars were positioned further upstream. The smaller noise reduction benefit observed with the bars of smaller width or length may be expected, as the narrower wake produced by the smaller bars induces a flow deficit over a reduced portion of the flap edge and may, therefore, have a weaker effect on the vortex system. Conversely, the similar noise reduction benefit obtained with bar\#2 and bar\#5 may indicate that once the bar is long enough for its wake to affect the full extent of the flap side edge, no additional noise reduction is obtained by further extending the bar length.

Although not as pronounced, the effects of bar width, length and placement on flap side edge noise shown above for the $39^{\circ}$ flap were also observed with the $29^{\circ}$ flap. The noise spectrum obtained with bar\#2 installed at $92 \%$ chord upstream of the $29^{\circ}$ flap is compared in Figure 11 to the spectrum obtained for the same bar configuration with the $39^{\circ}$ flap. The noise spectra obtained in the absence of the bar are also included. It is seen that even though flap side edge noise levels are 4 to $8 \mathrm{~dB}$ lower for the $29^{\circ}$ flap $^{7}$ than for the $39^{\circ}$ flap (because of the weaker vortex system associated with the smaller flap deflection angle), the noise levels produced by the $29^{\circ}$ flap are further reduced with the installation of the bar upstream of the side edge. 


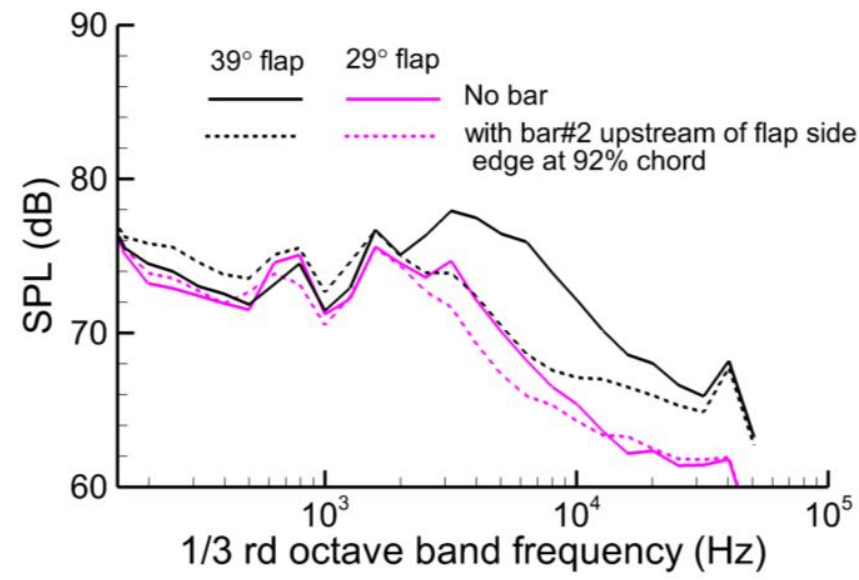

Figure 11. One-third octave band spectra from single microphone measurements for the $29^{\circ}$ and $39^{\circ}$ flaps with and without bar\# 2 installed upstream of the flap side edge at $92 \%$ chord; $M=0.17$.

Finally, the velocity dependence of the reduced flap side edge noise levels resulting from the wake/flap-side-edge interaction was examined for two bar streamwise stations, $92 \%$ and $36 \%$ chord. An optimum $6^{\text {th }}$ power level dependence on Mach number was found over the frequency range dominated by flap side edge noise (above $2 \mathrm{kHz}$ ). The spectra obtained for the $39^{\circ}$ flap with and without bar $\# 2$ positioned upstream of the flap side edge are presented in Figure 12. The spectral levels are normalized with the $6^{\text {th }}$ power of Mach number and the frequency is normalized in a Strouhal fashion where only Mach number is varied. It is seen that for each of the three test cases presented, the portion of the spectra shown earlier to be dominated by flap side edge noise (i.e., above $2 \mathrm{kHz}$ ) properly scales with the normalized velocity. However, between approximately $500 \mathrm{~Hz}$ and $2 \mathrm{kHz}$, although the levels scale with the $6^{\text {th }}$ power of Mach number, the spectra do not exhibit a frequency distribution that scales with velocity. Below $500 \mathrm{~Hz}$, noise levels do not follow any type of Mach number nor frequency scaling, with the exception of the broad, tonal like noise which is seen in the spectra (at $250 \mathrm{~Hz}$ normalized frequency) when the wake generator is positioned at $36 \%$ chord. The peak frequency of this broad "tone", which was shown earlier to develop as the bar is moved farther upstream from the flap edge, scales with the normalized frequency.
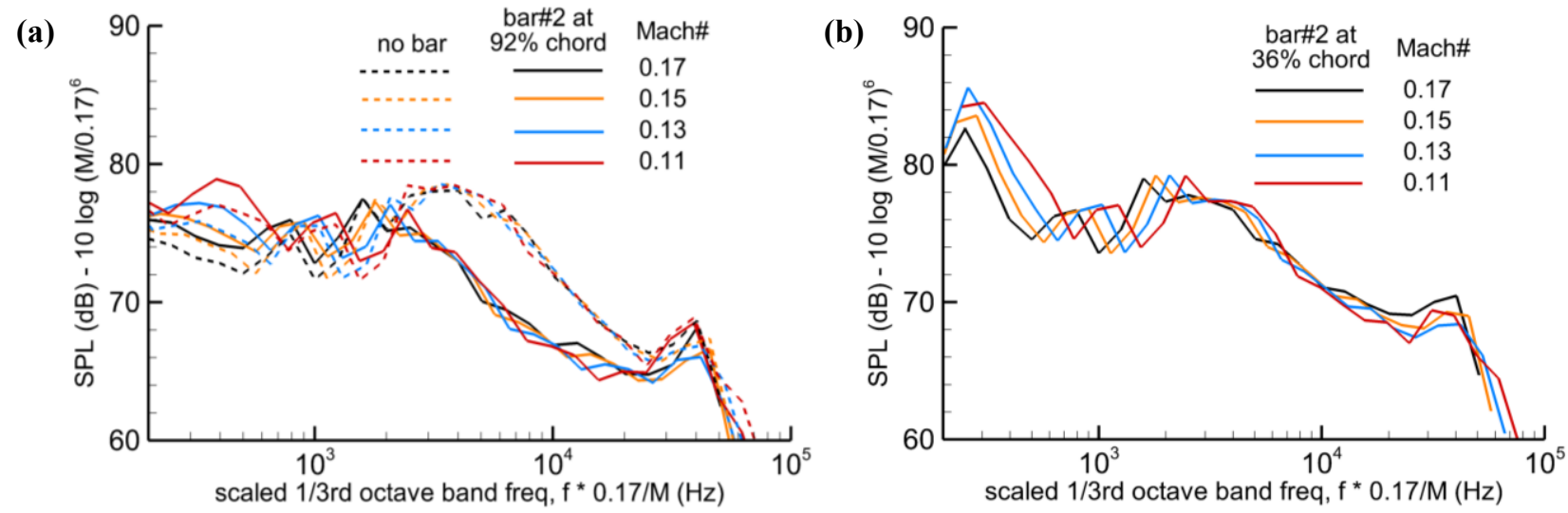

Figure 12. Effect of flow speed. Scaled one-third octave band spectra from single microphone measurements. (a) with and without bar\#2 positioned upstream of flap side edge at $92 \%$ chord; (b) with bar\#2 positioned upstream of flap side edge at $36 \%$ chord; $39^{\circ}$ flap. 


\subsection{Landing Gear Wake / Flap Interaction}

The noise resulting from the interaction of the wake produced by the simplified landing gear model with the halfspan flap is examined next. The landing gear model (wheels and truck removed) was mounted alternatively at $30 \%$ and 57\% chord (streamwise station of the landing gear's main strut), either inboard of the flap, upstream of the flap side edge, or outboard of the flap where the landing gear wake would have limited interaction with the flap. The single microphone noise spectra obtained for each of these landing gear placements are displayed in Figure 13 along with a sketch depicting the landing gear positions listed above. The flap is deployed at $39^{\circ}$, and the free stream flow Mach number is 0.17 . The noise spectrum obtained in the absence of the landing gear is also included. Figure 13 reveals that installation of the landing gear outboard of the flap leads to an increase in noise over the entire frequency range measured. It will be shown later that the large spectral peak near $1.25 \mathrm{kHz}$ is a result of wake interaction between the two landing gear side-braces. Removal of the downstream side-brace or of both side-braces leads, respectively, to either a large attenuation or elimination of this spectral peak.

At the 57\% chord streamwise station, placing the landing gear inboard of the flap (as compared to outboard) is observed to lead to a small increase in noise above $20 \mathrm{kHz}$ (frequency range of peak cove noise in the baseline case). The same increase is observed with the landing gear positioned upstream of the flap side edge. Placing the landing gear upstream of the flap side edge also results in lower noise than when the landing gear is positioned inboard or outboard of the flap, over a relatively broad frequency range $(2 \mathrm{kHz}$ to $20 \mathrm{kHz})$. Between approximately $5 \mathrm{and} 9 \mathrm{kHz}$, noise levels appear slightly lower than when the landing gear is not installed on the model. This seems to indicate that, as with the simple wake generators discussed in the previous section, the landing gear wake disrupts and weakens the flap side edge vortex system, decreasing flap side edge noise. Moving the landing gear further upstream on the airfoil, at $30 \%$ chord, either inboard of the flap or upstream of the side edge, is seen to lead to a small decrease in noise below $1 \mathrm{kHz}$ compared to the $57 \%$ chord streamwise location. Furthermore, above $20 \mathrm{kHz}$, noise levels are reduced to those obtained when the landing gear is positioned outboard of the flap at $57 \%$ chord.

The change in amplitude and frequency of the large spectral peak observed in Figure 13 between 1 and $2 \mathrm{kHz}$ for the different landing gear placements is believed to be associated with a corresponding change in local velocity in the landing gear region. The largest spectral peak (and highest peak frequency) occurs when the landing gear is positioned outboard of the flap (lowest adverse pressure gradient / highest local velocity) and the smallest spectral peak (and lowest peak frequency) occurs when the landing gear is positioned inboard and closest to the flap (greatest adverse pressure gradient / lowest local velocity).
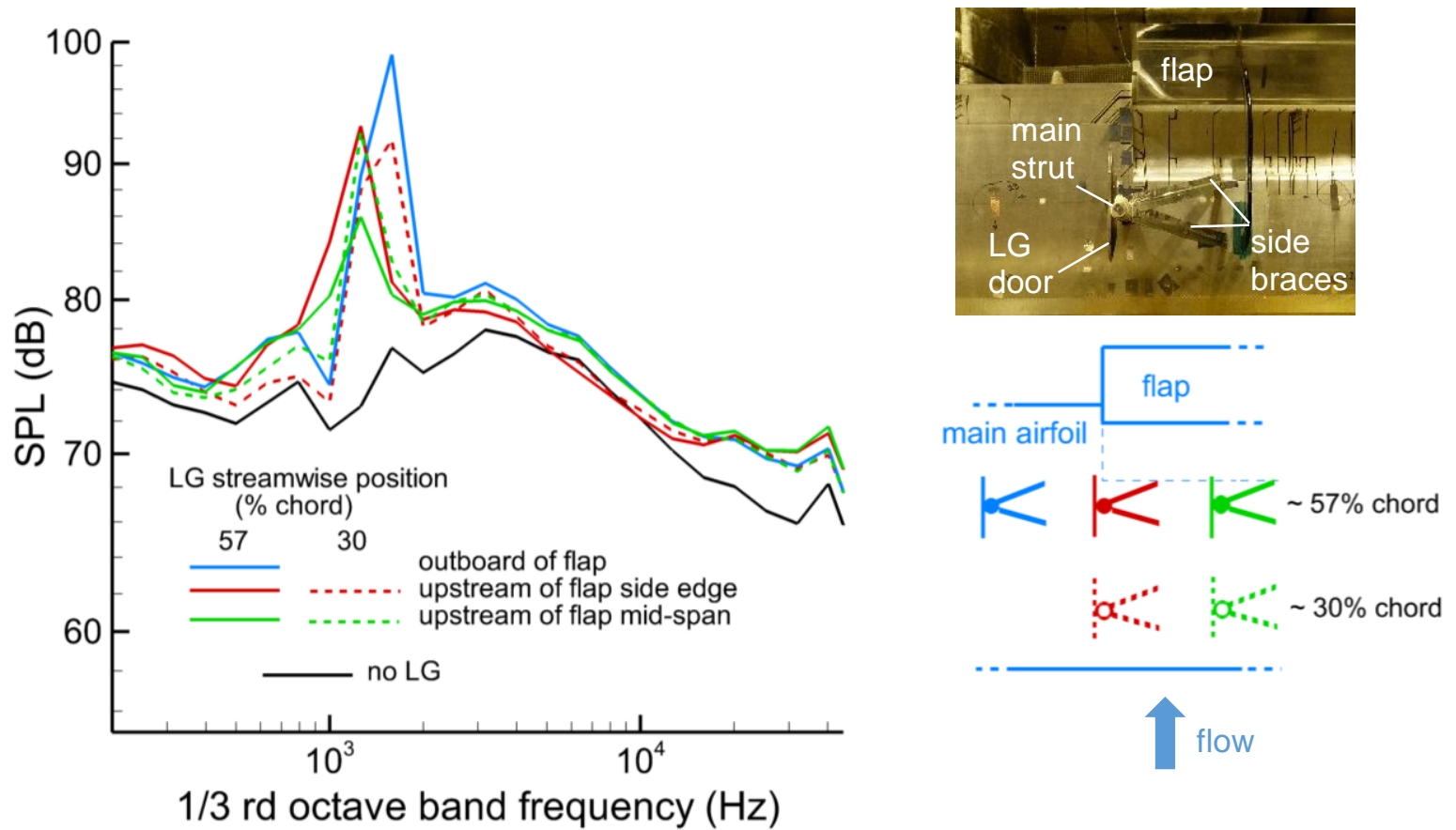

Figure 13. Effect of landing gear (LG) spanwise and streamwise placement. One-third octave band spectra from single microphone measurements. Landing gear wheels and truck are removed; $39^{\circ}$ flap; $M=0.17$. 
The effect of the landing gear placement on the noise radiating from the flap side edge and flap cove regions is illustrated below (in Figure 14) in the DAMAS noise source distribution maps obtained for the streamwise and spanwise positions discussed previously (outboard position not included). The 8 to $16 \mathrm{kHz}$ frequency range is shown. The DAMAS maps reveal the appearance of stronger noise sources in the flap cove region when the landing gear is inboard of the flap, and the presence of weaker noise sources at the flap side edge when the landing gear is positioned upstream of that edge. The corresponding $1 / 3^{\text {rd }}$ octave band spectra of the noise radiating from the flap side edge and flap cove regions are shown in Figure 15. These spectra are obtained from the DAMAS maps by summing the mean squared pressure over the corresponding regions. The portion of the landing gear region (defined in Figure 14) that overlaps the flap cove or flap side edge regions when the landing gear is at $57 \%$ chord is excluded from the mean squared pressure summations. The extent of the landing gear region was chosen somewhat arbitrarily. Broadening this region by two additional grid points on each side (bringing the downstream side of the region up to the leading edge of the flap) had very limited effect on the flap side edge noise spectra, but decreased the flap cove noise spectral levels by approximately $1 \mathrm{~dB}$ above $3.15 \mathrm{kHz}$.
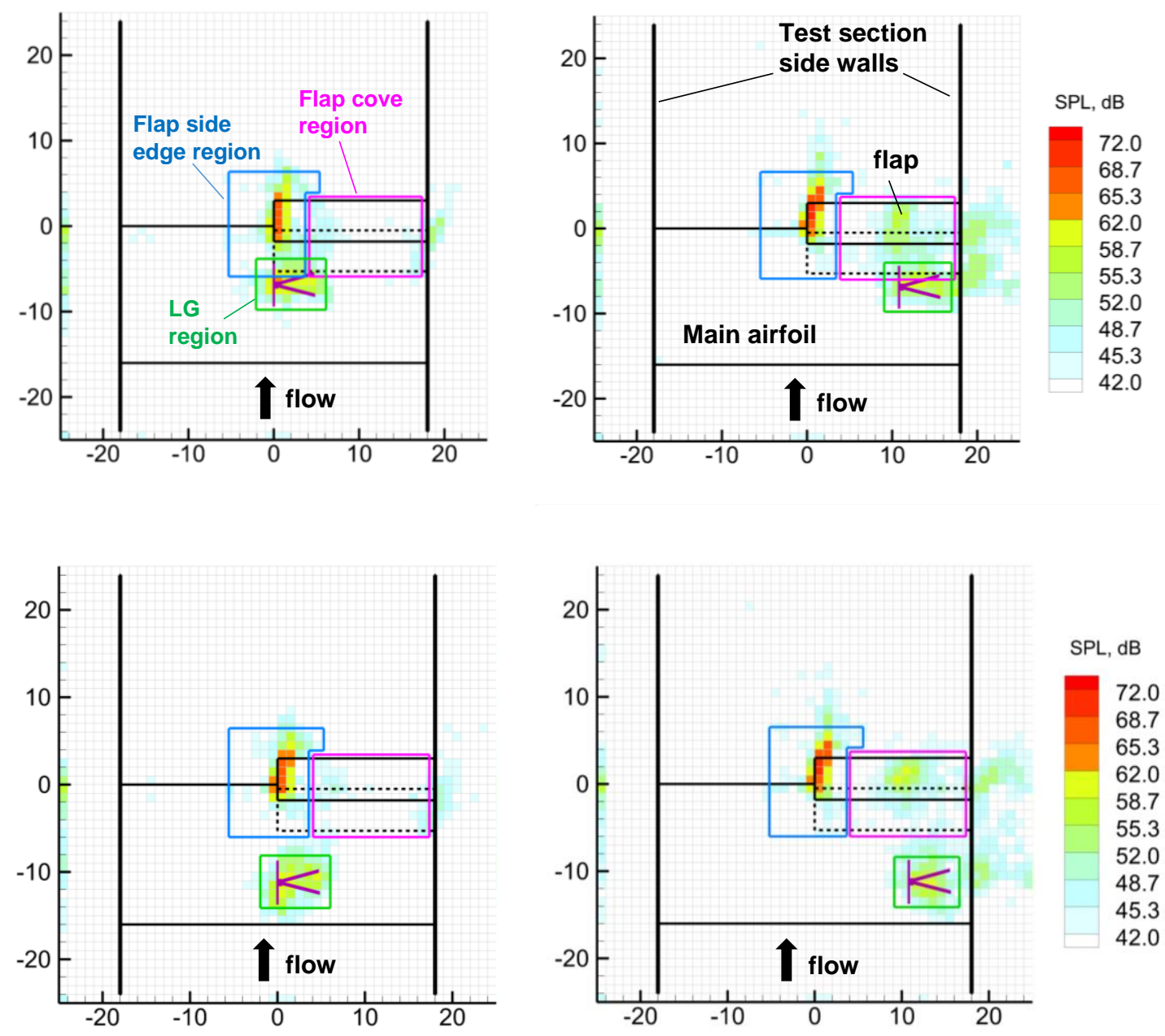

Figure 14. Effect of landing gear (LG) spanwise placement. Top two maps: LG at $57 \%$ chord; bottom two maps: LG at $30 \%$ chord. The LG wheels and truck are removed. DAMAS noise source distribution maps in summed one-third octave bands; $39^{\circ}$ flap; $M=0.17$; frequency range: $8 \mathrm{kHz}$ to $16 \mathrm{kHz}$. 
Figure 15(a) demonstrates that positioning the landing gear inboard of the flap does not affect the flap side edge noise spectral levels. They are the same as when the landing gear is removed, except for a 1 and $2 \mathrm{~dB}$ increase at 31.5 and $40 \mathrm{kHz}$, respectively, when the gear is closest to the flap. This landing gear position, however, leads to cove noise levels (Figure 15(b)) that are close to flap side edge noise levels below $3.15 \mathrm{kHz}$ (and at $40 \mathrm{kHz}$ ) and that are 2 to 8 $\mathrm{dB}$ higher than when the landing gear is aligned with the flap side edge. As with the bar models, positioning the landing gear upstream of the flap side edge leads to a reduction of flap side edge noise over a broad frequency range, namely 3.15 to $16 \mathrm{kHz}$, as shown in Figure 15(a). Finally, a change in the streamwise position of the landing gear is shown in Figure 15(b) to have little effect on flap cove noise but does affect the amount of flap side edge noise reduction achieved (Figure 15(a)). As observed with the bar models, increased noise reduction benefit is seen when the wake is generated closer to the flap edge.

(a)

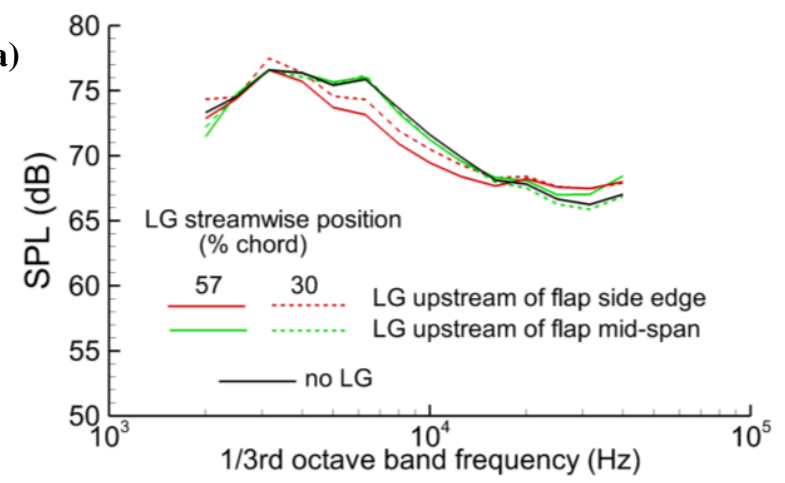

(b)

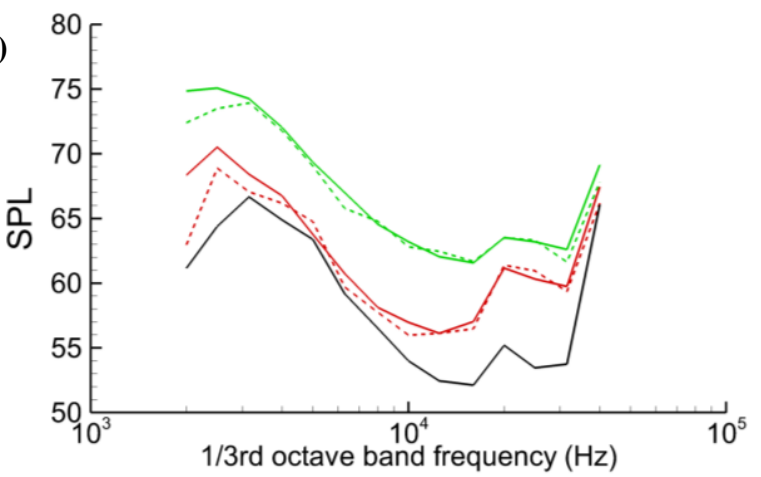

Figure 15. One-third octave band spectra of (a) noise radiating from the flap side edge region and (b) noise radiating from the flap cove region. Landing gear (LG) with wheels and truck removed; $39^{\circ}$ flap; $M=0.17$.

The effect of the landing gear side-braces on the radiated noise is examined next. The noise spectra obtained with the downstream side-brace removed and with both side-braces removed are compared, in Figure 16, to the spectra obtained with both side-braces in place. The landing gear is positioned upstream of the flap side edge (Figure 16(a)) and inboard of the flap (Figure 16(b)). The landing gear streamwise station is 57\% chord.
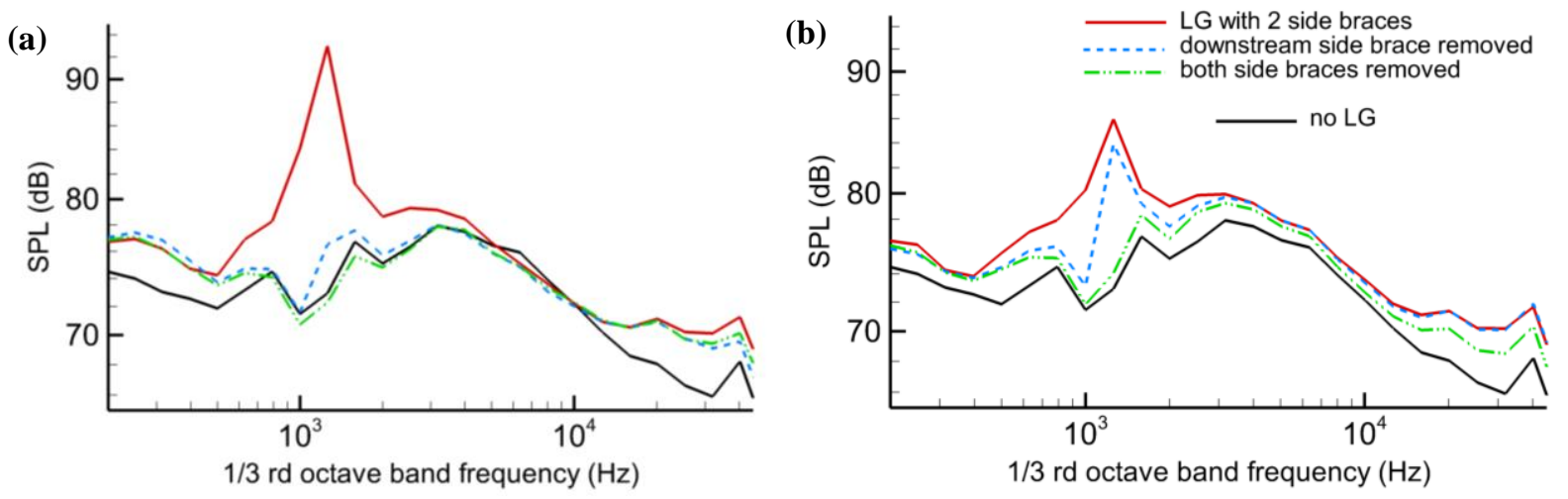

Figure 16. Effect of side-braces; (a) LG positioned upstream of the flap side edge; (b) LG positioned upstream of the flap mid-span. One-third octave band spectra from single microphone measurements; LG streamwise station: $57 \%$ chord; $39^{\circ}$ flap; $M=0.17$.

The broad spectral peak centered at $1.25 \mathrm{kHz}$ is seen to be attenuated with the removal of the downstream side-brace and eliminated without both side-braces. The noise attenuation associated with the removal of the side-braces occurs over a broad frequency range about the spectral peak (between $500 \mathrm{~Hz}$ and $5 \mathrm{kHz}$ when the landing gear is upstream of the flap side edge and between $315 \mathrm{~Hz}$ and $3.15 \mathrm{kHz}$ when the landing gear is inboard of the flap). Similar effects as in Figure 16(a) were observed with the landing gear positioned outboard of the flap or at 30\% chord (both upstream 
of the flap side edge and inboard of the flap). Removal of the downstream side-brace (only) led in each of these cases to a reduction of at least $15 \mathrm{~dB}$ and sometimes an elimination of the spectral peak. The large peak seen in the spectra when the landing gear is installed on the wing/flap model appears to be associated with noise radiating directly from vortex shedding from the side-braces, as well as with noise resulting from wake interaction between the two.

The effect of the landing gear side-braces on flap side edge, flap cove and landing gear noise are displayed in Figure 17 for the same two landing gear locations discussed in Figure 16. The noise spectra are obtained from DAMAS processing. The flap side edge, flap cove and landing gear regions are as defined in Figure 14. Figure 17(a) indicates that with the landing gear positioned upstream of the flap side edge, landing gear noise increases with the number of side-braces, while cove noise levels remain approximately unchanged with 0 or 1 brace and increase by approximately $2 \mathrm{~dB}$ with both braces. In contrast, flap side edge noise levels are seen to be further reduced (by up to $2 \mathrm{~dB}$ ) when one or both braces are installed. This improved reduction in flap side edge noise obtained with the presence of the sidebraces may be attributed to a broadening of the associated landing gear wake. The broader and/or more turbulent wake has a larger impact on the flap side edge vortex system responsible for the radiated noise.

With the landing gear positioned inboard of the flap (Figure 17(b)), flap cove noise levels slightly increase (by 0.5 to $2 \mathrm{~dB}$ ) with the number of side-braces, while flap side edge noise remains unchanged (except above $20 \mathrm{kHz}$ where the presence of 1 or 2 side-braces increased noise levels by $1 \mathrm{~dB}$ ). Landing gear noise levels are also lower overall and less sensitive to the presence of the second side-brace than when the gear is aligned with the flap side edge. This may be attributed to the lower local flow velocity expected upstream of the flap.

For the results presented in Figure 17, the portion of the landing gear region (defined in Figure 14) that overlaps the flap cove or flap side edge regions was again excluded from the mean squared pressure summations for the calculation of the flap cove and flap side edge noise spectra, respectively. Broadening the landing gear region by two additional grid points on each side slightly increased landing gear noise levels and decreased flap cove noise levels; however, the trends discussed above regarding the effect of the side-braces on noise remained the same.
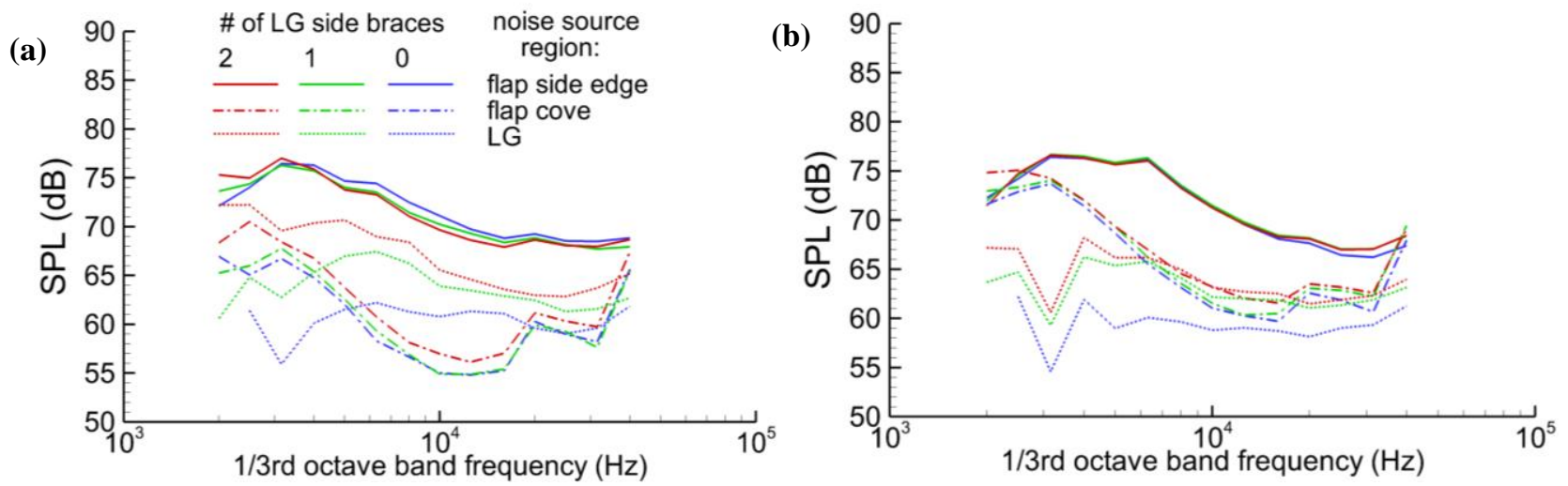

Figure 17. Effect of side-braces; (a) LG positioned upstream of the flap side edge; (b) LG positioned upstream of the flap mid-span. One-third octave band spectra from DAMAS processing; LG streamwise station: $57 \%$ chord; $39^{\circ}$ flap; $\mathrm{M}=\mathbf{0 . 1 7}$.

The effects of the landing gear spanwise station and side-braces on the noise radiating from the $29^{\circ}$ flap configuration are presented in Figure 18. One-third octave band spectra from single microphone measurements are shown. The amplitude of the large spectral peaks associated with noise from the side-braces is seen to be greater than for the $39^{\circ}$ flap configuration (Figure 16). This increase in noise from the side-braces is consistent with the higher local flow velocity expected with the smaller flap deflection angle. For the $39^{\circ}$ flap deflection case, noise levels between 2 and $20 \mathrm{kHz}$ (i.e., above the frequency range of the spectral peak) were shown to be reduced by up to $2 \mathrm{~dB}$ by positioning the landing gear upstream of the flap side edge instead of inboard of the flap, regardless of the number of side-braces. For the $29^{\circ}$ flap case, only when the landing gear is in a quieter configuration (i.e., downstream sidebrace removed) does the noise benefit of positioning the landing gear upstream of the flap side edge become significant. Approximately $1 \mathrm{~dB}$ in noise reduction occurs above $2 \mathrm{kHz}$ when the downstream side-brace is removed versus $1 \mathrm{~dB}$ above $6 \mathrm{kHz}$ with both side-braces in place. 
It was shown in this section that positioning the landing gear upstream of the flap side edge leads to reduced flap side edge noise and to cove noise levels significantly lower than when the landing gear is positioned inboard of the flap. However, when searching for the quietest landing gear placement, direct noise radiation from the landing gear has to be taken into consideration, as it may be exposed to a higher local flow velocity upstream of the flap side edge than inboard of the flap.

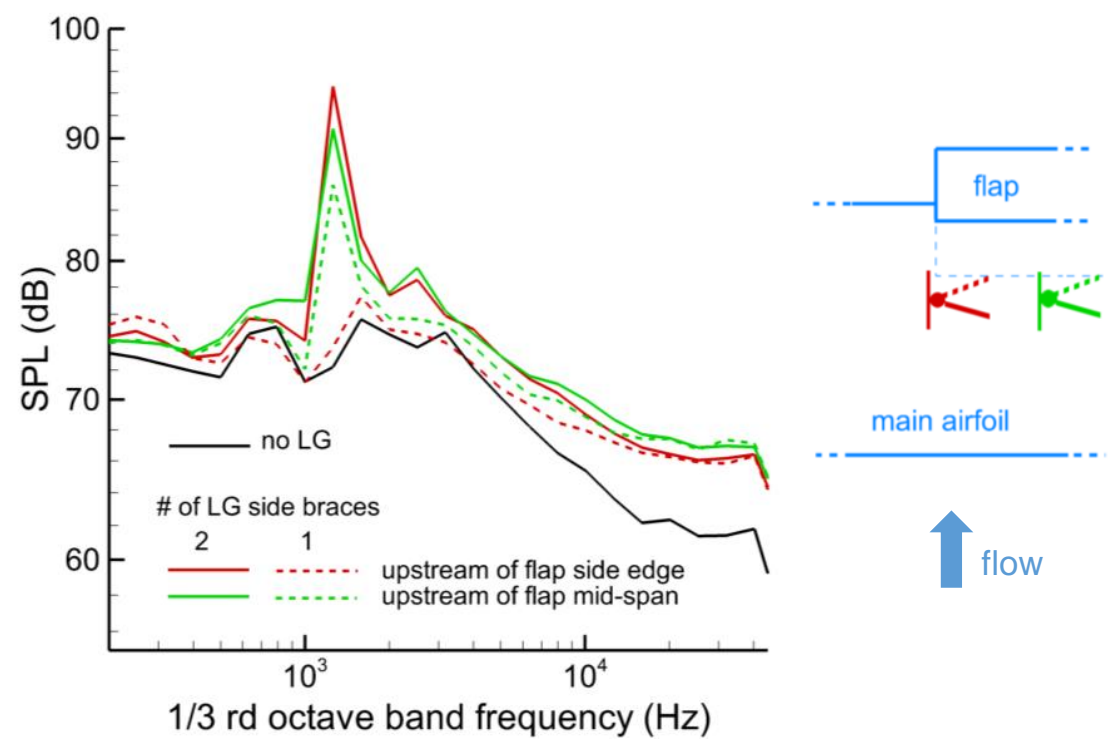

Figure 18. Effects of side-braces and landing gear (LG) spanwise station for the $29^{\circ}$ flap. One-third octave band spectra from single microphone measurements. LG streamwise station: $57 \%$ chord; $M=0.17$.

\section{Conclusion}

The effects on radiated noise of the interaction of a wake with a half-span flap were examined. The wake was generated by simple bars or by a simplified landing gear model.

It was shown that significant reduction in flap side edge noise can be achieved with a bar positioned upstream of the flap side edge. Noise reduction levels were found to increase with increased bar width and length, and with the bar proximity to the flap edge. The reduction in flap side edge noise is attributed to a weakening of the vortex system responsible for the radiated noise as the bar wake introduces a velocity deficit and modifies the instabilities in the vicinity of the flap side edge. The decreased flap side edge noise levels resulting from the wake/flap-side-edge interaction were also shown to follow a $6^{\text {th }}$ power level dependence on Mach number.

Positioning the bars inboard of the flap was found not to affect the flap side edge noise but led to a significant increase in cove noise. The increased cove noise levels also showed little sensitivity to changes in the bar streamwise station, indicating that the broadening of the wake that occurs when the bar is moved further upstream of the flap might be compensated for by a reduction in the intensity of the vortices entering the flap cove region.

As with the bar models, positioning the landing gear (truck and wheels removed) upstream of the flap side edge was observed to lead to a reduction in flap side edge noise over a broad frequency range with an increased noise reduction benefit obtained with the wake generated closer to the flap edge. Positioning the landing gear inboard of the flap showed little effect on flap side edge noise. However, such placement led to a significant increase in cove noise, which was not affected by changes in the landing gear streamwise position. The installation of the landing gear model on the main airfoil was associated with an increase in noise over the entire measured frequency range and the generation of a broad and large spectral peak in the low frequency range. This spectral peak was found to be associated with the noise radiating from the two landing gear side-braces and particularly from wake interaction between the two side-braces. Removal of the downstream side-brace and of both side-braces led, respectively, to a large attenuation and elimination of this spectral peak. Examination of the effects of the landing gear side-braces on flap side edge and 
cove noise revealed that cove noise increases with the number $(0,1$ or 2$)$ of side-braces installed (landing gear positioned inboard of the flap or aligned with the side edge), while flap side edge noise is slightly more reduced with the side-braces in place (landing gear upstream of flap side edge).

Although positioning the landing gear upstream of the flap side edge led to decreased flap side edge noise and to cove noise levels significantly lower than when the landing gear is positioned inboard of the flap, direct noise radiation from the landing gear needs to be taken into account, as the landing gear is likely to be exposed to a higher local flow velocity upstream of the flap side edge than inboard of the flap. However, for a sufficiently quiet landing gear design, the gear should be positioned upstream of the flap side edge to produce the least noise from the gear/flap system.

\section{Acknowledgments}

The authors wish to gratefully acknowledge Jaye A. Moen and Dennis H. Kuchta for their support in this experiment.

\section{References}

[1] Oerlemans, S. and Pott-Pollenske, M., “An Experimental Study of Landing Gear Wake/Flap Interaction Noise," AIAA paper 2004-2886.

[2] Khorrami, M., Mineck, R, Yao, C. and Jenkins, L., “A Comparative Study of Simulated and Measured Gear-Flap Flow Interaction,” AIAA paper 2015-2989.

[3] Brooks, T. and Humphreys, W., "Flap Edge Aeroacoustics Measurements and Predictions," Journal of Sound and Vibration, Vol. 261, pp.31-74, 2003.

[4] Hutcheson, F. and Brooks, T., "Effects of Angle of Attack and Velocity on Trailing Edge Noise Determined Using Microphone Array Measurements," International Journal of Aeroacoustics, Vol.5, number 1, pp. 39-66, 2006.

[5] Hutcheson, F. and Brooks, T., "Noise Radiation from Single and Multiple Rod Configurations," $12^{\text {th }}$ AIAA/CEAS Aeroacoustics Conference, AIAA paper no. 2006-2629, Cambridge, MA, May 2006.

[6] Brooks, T. and Humphreys, W., "A deconvolution approach for the mapping of acoustic sources (DAMAS) determined from phased microphone arrays," Journal of Sound and Vibration, Vol. 294, pp. 856-879, 2006.

[7] Hutcheson, F. and Brooks, T., "Noise Radiation from a Continuous Mold-Line Link Flap Configuration," International Journal of Aeroacoustics, Vol. 11, no. 5\&6, 2012.

[8] Khorrami, M., Singer, B. and Takallu, M., "Analysis of Flap Side Edge Flow Field for Identification and Modeling of Possible Noise Sources,” SAE Paper 971917, May 1997.

[9] Balakrishnan, S. and Shariff, K., "Detached Eddy Simulation of Flap Side-Edge Flow," AIAA paper 2016-1074. 